\title{
ARCWISE CONNECTEDNESS OF SEMIAPOSYNDETIC PLANE CONTINUA
}

\author{
BY \\ CHARLES L. HAGOPIAN
}

\begin{abstract}
In a recent paper, the author proved that if a compact plane continuum $M$ contains a finite point set $F$ such that, for each point $x$ in $M-F, M$ is semilocally-connected and not aposyndetic at $x$, then $M$ is arcwise connected. The primary purpose of this paper is to generalize that theorem. Semiaposyndesis, a generalization of semi-local-connectedness, is defined and arcwise connectedness is established for certain semiaposyndetic plane continua.
\end{abstract}

A compact plane continuum which is semi-locally-connected may fail to be arcwise connected [9]. F. Burton Jones has conjectured that although the arcwise connectedness theorem for locally connected continua does not extend to semilocally-connected spaces, there are general arcwise connectedness theorems for nonlocally connected plane continua [7]. According to Jones, a continuum $M$ is said to be aposyndetic at a point $p$ of $M$ with respect to a point $q$ of $M-\{p\}$ if there exist an open set $U$ and a continuum $H$ in $M$ such that $p \in U \subset H \subset M-\{q\}$. A continuum $M$ is said to be aposyndetic at a point $p$ if for each point $q$ in $M-\{p\}$, $M$ is aposyndetic at $p$ with respect to $q$. Recent results indicate that if a compact plane continuum $M$ contains a finite point set $F$ such that, for each point $x$ in $M-F, M$ is semi-locally-connected and not aposyndetic at $x$, then $M$ is arcwise connected [1, Theorem 9]. In fact, plane continua with these properties are hereditarily arcwise connected [3, Theorem 1]. Note that since $M$ is semilocally-connected at each point of $M-F$ and is not aposyndetic at any point of $M-F$, for each point $x$ of $M-F$ there exists a point $y$ of $F$ such that $M$ is not aposyndetic at $x$ with respect to $y$ [6, Theorem 0$]$.

There are many non-locally-connected arcwise connected plane continua which do not have the properties stated in the hypothesis of this theorem. For example the Cantor cone (the upper semicontinuous decomposition of the topological product of the unit interval $[0,1]$ and the Cantor discontinuum $C$ in which the set $0 \times C$ is a point) is arcwise connected and fails to have the "semi-local-connectedness at all but a finite number of points" property.

Definition. Let $p$ be a point of a continuum $M . M$ is said to be semiaposyndetic at $p$ if for each point $q$ of $M-\{p\}, M$ is aposyndetic at $p$ with respect to $q$ or $M$ is

Received by the editors April 16, 1970 and, in revised form, December 1, 1970.

AMS 1969 subject classifications. Primary 5455, 5475; Secondary 5422.

Key words and phrases. Semiaposyndesis, semi-local-connectedness, aposyndesis, arcwise connected continua, folded complementary domain.

Copyright (C) 1971, American Mathematical Society 
aposyndetic at $q$ with respect to $p$. A continuum is said to be semiaposyndetic if it is semiaposyndetic at each of its points.

In this paper the following theorem is established. If a compact plane continuum $M$ contains a finite point set $F$ such that, for each point $x$ in $M-F, M$ is semiaposyndetic at $x$ and is not aposyndetic at $x$ with respect to some point of $F$, then $M$ is arcwise connected. Since a continuum $M$ is semi-locally-connected at a point $p$ of $M$ only if $M$ is semiaposyndetic at $p$, this is a generalization of the preceding theorem. Note that the Cantor cone has the properties stated in this more general theorem.

Throughout this paper $S$ is the set of points of a simple closed surface (that is, a 2-sphere). For definitions of unfamiliar terms and phrases see [8] and [10].

THEOREM 1. Suppose that $M$ is a continuum in $S$ and the set of points of $M$ at which $M$ is not semiaposyndetic is countable. Then if $x$ and $y$ are distinct points of $M$ and $M$ is not aposyndetic at $x$ with respect to $y$, there exists an arc $J$ in $M$ with extremities $x$ and $y$ such that, for each point $z$ of $J-\{y\}, M$ is not aposyndetic at $z$ with respect to $y$.

Proof. Since $M$ is not aposyndetic at $x$ with respect to $y$, the set $S-M$ is folded [5, Theorem 2]. In fact, $S-M$ is folded around $x$ with respect to $y$ [2]. It follows that there exist two monotone descending sequences of circular regions $U_{1}, U_{2}, U_{3}, \ldots$ and $V_{1}, V_{2}, V_{3}, \ldots$ in $S$ centered on and converging to $x$ and $y$ respectively, such that $\mathrm{Cl} U_{1} \cap \mathrm{Cl} V_{1}=\varnothing\left(\mathrm{Cl} U_{1}\right.$ is the closure of $\left.U_{1}\right)$, and there exists a sequence of mutually exclusive sets $X_{1}, X_{2}, X_{3}, \ldots$ in $S-M$ having the following properties. For each positive integer $i$, the set $X_{i}$ is the union of two intersecting arc-segments (open arcs) $I_{i}$ and $T_{i}$ such that (1) $I_{i} \cap T_{i}$ is connected, (2) $I_{i}$ is contained in Bd $U_{i}$ (Bd $U_{i}$ is the boundary of $U_{i}$ ) and has endpoints $a_{i}$ and $b_{i}$ in $M$, (3) $T_{i}$ is contained in $S-\mathrm{Cl}\left(V_{i} \cup U_{i+1}\right)$ and has two distinct endpoints in $\mathrm{Bd} V_{i}-M$, and (4) $T_{i} \cup \operatorname{Bd} V_{i}$ contains a simple closed curve $S_{i}$ which separates $a_{i}$ from the set $\left\{b_{i}, x, y\right\}$ in $S$. Note that for each positive integer $i, \mathrm{Cl} T_{i}$ separates the $a_{i}$-component of $M-V_{i}$ from the $x$-component of $M-V_{i}$ in $S-V_{i}$.

For each positive integer $i$, let $A_{i}$ be the $a_{i}$-component of $M-V_{i}$. Let $A$ be the limiting set of $A_{1}, A_{2}, A_{3}, \ldots$ The set $A$ is a subcontinuum of $M$ which contains $x$ and $y\left[8\right.$, Theorem 58, p. 23]. There exists a subsequence of $A_{1}, A_{2}, A_{3}, \ldots$ which converges to a continuum containing $x$ and $y$ in $M$ [8, Theorem 59, p. 24]. Assume without loss of generality that $A_{1}, A_{2}, \dot{A}_{3}, \ldots$ converges to $A$. Note that for each point $z$ of $A-\{y\}, M$ is not aposyndetic at $z$ with respect to $y$. For each positive integer $i$, define $Y_{i}$ to be the $x$-component of $A-V_{i}$. Let $Y$ be the limiting set of $Y_{1}, Y_{2}, Y_{3}, \ldots$ The set $Y$ is a subcontinuum of $A$ which contains $x$ and $y$.

Assume that $Y$ is not locally connected. It follows that $Y$ is not connected im kleinen at some point $v$ in $Y-\{x, y\}$. There exist two circular regions $T$ and $W$ in $S$ centered on $v$ such that (1) $T \supset \mathrm{Cl} W$, (2) $\{x, y\} \cap \mathrm{Cl} T=\varnothing$, and (3) there exists 
a sequence of mutually exclusive continua $H_{1}, H_{2}, H_{3}, \ldots$ in $Y \cap(\mathrm{Cl} T-W)$ such that each continuum meets both $\mathrm{Bd} T$ and $\mathrm{Bd} W$ and the sequence converges to a continuum $Z$ [8, Theorem 66 (Proof), p. 124]. It is not necessary here to insist that $H_{1}, H_{2}, H_{3}, \ldots$ be in different components of $Y \cap(\mathrm{Cl} T-W)$, since $Y$ is a continuum of convergence of $M$. Let $q$ be a point of $Z$ which is in $T-\mathrm{Cl} W$. Let $q_{1}, q_{2}, q_{3}, \ldots$ be a sequence of points which converges to $q$ such that, for each positive integer $n, q_{n} \in H_{n} \cap(T-\mathrm{Cl} W)$. Let $Q_{1}, Q_{2}, Q_{3}, \ldots$ be a sequence of mutually exclusive circular regions in $S$ which converges to $q$ such that, for each positive integer $n$, the region $Q_{n}$ is centered on $q_{n}$ and $\mathrm{Cl} Q_{n}$ is contained in $T-\mathrm{Cl} W$. For each positive integer $n$, there exists an integer $i$ such that $Y_{i} \cap Q_{n} \neq \varnothing$. It follows that there exists a sequence of mutally exclusive continua $J_{1}, J_{2}, J_{3}, \ldots$ in $Y \cap(\mathrm{Cl} T-W)$ which converges to a continuum $I$ containing $q$ in $Y$ such that, for each positive integer $n, J_{n}$ meets $\mathrm{Bd}(T-W)$ and there exists an integer $i$ such that $J_{n} \subset Y_{i}$. Note that $I$ meets $\mathrm{Bd}(T-W)$. Since $M$ is semiaposyndetic at all except at most countably many of its points, there exists a point $p$ and two circular regions $R$ and $E(R \supset \mathrm{Cl} E)$ centered on $q$ in $T-W$ such that (1) the point $p$ is in $(R-\mathrm{Cl} E) \cap I$, (2) $M$ is semiaposyndetic at $p$, and (3) there exists a sequence of mutually exclusive continua $F_{1}, F_{2}, F_{3}, \ldots$ such that, for each positive integer $n$, there exists an integer $i$ such that $F_{n}$ is in $J_{i} \cap(\mathrm{Cl} R-E), F_{n}$ meets both $\mathrm{Bd} R$ and $\mathrm{Bd} E$, and the sequence $F_{1}, F_{2}, F_{3}, \ldots$ converges to a continuum in $Y$ which contains $p$.

Assume without loss of generality that the sequence $F_{1}, F_{2}, F_{3}, \ldots$ is such that for each positive integer $n$, there exist arc-segments $R_{n}$ and $E_{n}$ such that (1) $R_{n} \subset \mathrm{Bd} R$, (2) $E_{n} \subset \mathrm{Bd} E$, and (3) each arc-segment meets $F_{1}, F_{2}, F_{3}, \ldots$ only in $F_{2 n}$ and has one endpoint in $F_{2 n-1}$ and the other endpoint in $F_{2 n+1}$. Let $p_{1}, p_{2}, p_{3}, \ldots$ be a sequence of points which converges to $p$ such that, for each positive integer $n$, the point $p_{n}$ is in $F_{2 n} \cap(R-\mathrm{Cl} E)$. Let $P_{1}, P_{2}, P_{3}, \ldots$ be a sequence of circular regions in $S$ such that, for each positive integer $n$, the region $P_{n}$ is centered on $P_{n}$ and $\mathrm{Cl} P_{n}$ does not meet $F_{2 n-1} \cup F_{2 n+1} \cup R_{n} \cup E_{n}$. Note that the regions of the sequence $P_{1}, P_{2}, P_{3}, \ldots$ are mutually exclusive, since $F_{2 n-1} \cup F_{2 n+1} \cup R_{n} \cup E_{n}$ separates $\mathrm{Cl} P_{n}$ from $\mathrm{Cl} P_{i}$, for $i \neq n$ [8, Theorem 28, p. 156], and converge to $p$.

There exist subsequences $V_{n_{1}}, V_{n_{2}}, V_{n_{3}}, \ldots$ of $V_{1}, V_{2}, V_{3}, \ldots$ and $A_{n_{1}}, A_{n_{2}}, A_{n_{3}}, \ldots$ of $A_{1}, A_{2}, A_{3}, \ldots$ such that $\mathrm{Cl} V_{n_{1}} \cap \mathrm{Cl} R=\varnothing$ and for each positive integer $k$, the set $F_{2 k-1} \cup F_{2 k} \cup F_{2 k+1} \cup F_{2 k+2}$ is in the $x$-component of $Y-V_{n_{k}}$ and $A_{n_{k}}$ meets $P_{k}$ and $P_{k+1}$.

For each positive integer $k$, let $e_{k}$ be a point of $A_{n_{k}} \cap \mathrm{Bd} P_{k}$ and let $g_{k}$ be a point of $A_{n_{k}} \cap \mathrm{Bd} P_{k+1}$. For each positive integer $k$, there exists an arc-segment $B_{k}$ belonging to $\left\{E_{k}, R_{k}\right\}$ such that $B_{k} \cup\left(x\right.$-component of $Y-V_{n_{k}}$ ) separates $e_{k}$ from $g_{k}$ in $S$ [8, Theorem 20, p. 173]. Let $s$ be a limit point of the sequence of arcsegments $B_{1}, B_{2}, B_{3}, \ldots$

$M$ is not aposyndetic at $s$ with respect to $p$. To see this assume $M$ is aposyndetic at $s$ with respect to $p$. It follows that there exist mutually exclusive circular regions 
$U, V$ in $S$ and a continuum $H$ in $M$ such that $p$ is in $U$ and $s \in M \cap V \subset H \subset S-\mathrm{Cl} U$. There exists a positive integer $k$ such that $P_{k} \cup P_{k+1} \subset U$ and $\mathrm{Cl} B_{k} \subset V$. Let $K$ denote the $x$-component of $Y-V_{n_{k}}$. Define $D$ to be the complementary domain of $K \cup B_{k}$ which contains $e_{k}$. Since Cl $T_{n_{k}}$ separates $A_{n_{k}}$ from $K$ in $S-V_{n_{k}}, T_{n_{k}}$ meets both Bd $P_{k} \cap D$ and Bd $P_{k+1} \cap(S-(K \cup D))$. Let $z$ be a point of $D \cap \operatorname{Bd} P_{k} \cap T_{n_{k}}$. The boundary of the component of $D \cap U$ which contains $z$ is contained in $K \cup \operatorname{Bd} U$. It follows that $T_{n_{k}}$ meets $D \cap \operatorname{Bd} U$. Let $w$ be a point of $(S-D) \cap$ Bd $P_{k+1} \cap T_{n_{k}}$. The boundary of the w-component of $U \cap\left(S-\left(K \cup B_{k}\right)\right)$ is contained in $K \cup \operatorname{Bd} U$. Therefore $T_{n_{k}}$ must also meet $(S-(K \cup D)) \cap \operatorname{Bd} U$. Assume without loss of generality that $T_{n_{k}}$ meets $D \cap \mathrm{Bd} U$ before it meets $(S-(K \cup D)) \cap \mathrm{Bd} U$ with respect to the linear order on $T_{n_{k}}$. Let $f$ be the first point of $T_{n_{k}} \cap(S-(K \cup D)) \cap \operatorname{Bd} U$ and let $h$ be the last point of $T_{n_{k}} \cap D \cap \operatorname{Bd} U$ which precedes $f$ with respect to the order on $T_{n_{k}}$. Let $X$ denote the subarc of $T_{n_{k}}$ which has endpoints $h$ and $f$. Note that $X \cap \mathrm{Cl} U=\{h, f\}$. Let $C_{1}$ and $C_{2}$ be the mutually exclusive arc-segments in $\mathrm{Bd} U$ which have endpoints $h$ and $f$. Since $K \cup B_{k}$ separates $h$ from $f$ in $S$, the set $\left(K \cup B_{k}\right)-U$ contains a continuum $C$ which separates $h$ from $f$ in $S-U$ [8, Theorem 27, p. 177]. For $i=1$ and 2, there exists a point $c_{i}$ in $C \cap C_{i}$. The points $c_{1}$ and $c_{2}$ are contained in distinct components of $C-B_{k}\left[8\right.$, Theorem 28, p. 156]. For $i=1$ and 2, let $d_{i}$ be a point of $\mathrm{Cl} B_{k} \cap$ $\mathrm{Cl}\left(c_{i}\right.$-component of $\left.C-B_{k}\right)$. The set ( $\theta$-curve) $X \cup \mathrm{Bd} U$ separates $d_{1}$ from $d_{2}$ in $S$ [8, Theorem 28, p. 156]. $X \cup \operatorname{Bd} U$ is contained in $S-H$ and the set $\left\{d_{1}, d_{2}\right\}$ is contained in $H$. Since $H$ is connected, this is a contradiction.

Suppose that $M$ is aposyndetic at $p$ with respect to $s$. There exist mutually exclusive circular regions $Q$ and $O$ in $S$ and a continuum $F$ in $M$ such that $s$ is in $Q$ and $p \in M \cap O \subset F \subset S-Q$. There exists a positive integer $j$ such that $P_{j} \cup P_{j+1} \subset O$ and $B_{j} \subset Q$. Let $L$ be the set $B_{j} \cup\left(x\right.$-component of $\left.Y-V_{n_{j}}\right)$. Either the complementary domain $G$ of $L$ which contains $e_{j}$ does not meet $\mathrm{Cl} V_{n_{j}}$ or the complementary domain of $L$ which contains $g_{j}$ does not meet $\mathrm{Cl} V_{n j}$ since $V_{n j}$ is a connected subset of $S-L$. Assume without loss of generality that $G \cap \mathrm{Cl} V_{n_{j}}=\varnothing$. Let $r$ be a limit point of the $e_{j}$-component of $\left(\mathrm{Bd} P_{j} \cap G\right)$ which is not in $G$. Since $r$ is contained in the $x$-component of $Y-V_{n_{j}}$, the simple closed curve $S_{n_{j}}$ separates $e_{j}$ from $r$ in $S$. Furthermore $B_{j} \cup\left(T_{n} \cap G\right)$ separates $r$ from $e_{j}$ in $S$. This follows from the contrapositive of [8, Theorem 20, p. 173] and the observations that, if $B$ is an arc in $B_{j}$ that contains $\mathrm{Cl} S_{n,} \cap \mathrm{Bd} G$, then (1) $B \cup\left(S_{n,} \cap G\right)$ and $B \cup\left(S_{n,} \cap(S-G)\right)$ are closed and compact point sets with a connected intersection and their union separates $r$ from $e_{j}$ in $S$, (2) $B \cup\left(S_{n_{j}} \cap(S-G)\right)$ does not separate $r$ from $e_{j}$ in $S$, and (3) $B_{j} \cup\left(T_{n_{j}} \cap G\right) \supset B \cup\left(S_{n_{j}} \cap G\right)$. The continuum $F$ contains the set $\left\{r, e_{j}\right\}$ and does not meet $B_{j} \cup T_{n_{j}}$. This is clearly impossible. Hence $M$ is not aposyndetic. at $p$ with respect to $s$. But this contradicts the fact that $M$ is semiaposyndetic at $p$. It follows that $Y$ is a locally connected subcontinuum of $A$ and there exists an $\operatorname{arc} J$ lying in $Y$ with endpoints $x$ and $y$ [8, Theorem 13, p. 91]. Since $J \subset Y \subset A$, for each point $z$ of $J-\{y\}, M$ is not aposyndetic at $z$ with res pect to $y$. 
THEOREM 2. If a compact plane continuum $M$ contains a finite point set $F$ such that, for each point $x$ in $M-F, M$ is semiaposyndetic at $x$ and there exists a point $y$ of $F$ such that $M$ is not aposyndetic at $x$ with respect to $y$, then $M$ is arcwise connected.

Proof. For each point $y$ of $F$, let $L_{y}$ be the set consisting of $y$ and all points $x$ of $M-\{y\}$ such that $M$ is not aposyndetic at $x$ with respect to $y$. For each point $y$ of $F$, the set $L_{y}$ is a continuum [6, Theorem 3] and is (according to Theorem 1) arcwise connected. $M=\bigcup_{y \in F} L_{y}$. The continuum $M$ being the union of a finite number of arcwise connected continua is arcwise connected.

\section{BIBLIOGRAPHY}

1. C. L. Hagopian, Concerning arcwise connectedness and the existence of simple closed curves in plane continua, Trans. Amer. Math. Soc. 147 (1970), 389-402. MR 40 \#8030.

2. - A cut point theorem for plane continua, Duke Math. J. (to appear).

3. - Concerning the cyclic connectivity of plane continua, Michigan Math. J. (to appear).

4. F. B. Jones, Aposyndetic continua and certain boundary problems, Amer. J. Math. 63 (1941), 545-553. MR 3, 59.

5. - A characterization of a semi-locally-connected plane continuum, Bull. Amer. Math. Soc. 53 (1947), 170-175. MR 8, 397.

6. - Concerning non-aposyndetic continua, Amer. J. Math. 70 (1948), 403-413. MR 9, 606 .

7. - Problems in the plane, Summary of Lectures and Seminars, Summer Institute on Set Theoretic Topology, Madison, Wisconsin 1955; revised 1957, pp. 70-71.

8. R. L. Moore, Foundations of point set theory, rev. ed., Amer. Math. Soc. Colloq. Publ., vol. 13, Amer. Math. Soc., Providence, R. I., 1962.

9. G. T. Whyburn, Semi-locally connected sets, Amer. J. Math. 61 (1939), 733-749. MR 1, 31.

10. —, Analytic topology, rev. ed., Amer. Math. Soc. Colloq. Publ., vol. 28, Amer. Math. Soc., Providence, R. I., 1963. MR 32 \#425.

\section{Sacramento State College, \\ SaCramento, California 95822}

